\title{
Exploring the State Space Structure of Multiple Spins via Modular Tensor Diagram Approach: Going beyond the Exciton Pair State
}

Yunshu Tan, Guohua Tao*

School of Advanced Materials, Peking University Shenzhen Graduate School, Shenzhen 518055,

China; Shenzhen Key Laboratory of

New Energy Materials by Design, Peking University, Shenzhen 518055, China

\section{Supplementary information}

1. Function S1a-S1bl refer to the 64 exciton trimers spin base functions. The functions are classified according to different subspaces.

1 singlet-singlet-singlet trimer(SSS):

$\phi_{1}=S^{0} S^{0} S^{0}=\frac{1}{2 \sqrt{2}}(\alpha \beta-\beta \alpha)(\alpha \beta-\beta \alpha)(\alpha \beta-\beta \alpha)$

9 singlet-singlet-triplet trimers(SST):

$\phi_{2}=S^{0} S^{0} T^{+1}=\frac{1}{2}(\alpha \beta-\beta \alpha)(\alpha \beta-\beta \alpha) \alpha \alpha$

$\phi_{3}=S^{0} S^{0} T^{0}=\frac{1}{2 \sqrt{2}}(\alpha \beta-\beta \alpha)(\alpha \beta-\beta \alpha)(\alpha \beta+\beta \alpha)$

$\phi_{4}=S^{0} S^{0} T^{-1}=\frac{1}{2}(\alpha \beta-\beta \alpha)(\alpha \beta-\beta \alpha) \beta \beta$

$\phi_{5}=S^{0} T^{+1} S^{0}=\frac{1}{2}(\alpha \beta-\beta \alpha) \alpha \alpha(\alpha \beta-\beta \alpha)$

$\phi_{6}=S^{0} \mathrm{~T}^{0} S^{0}=\frac{1}{2 \sqrt{2}}(\alpha \beta-\beta \alpha)(\alpha \beta+\beta \alpha)(\alpha \beta-\beta \alpha)$

$\phi_{7}=S^{0} T^{-1} S^{0}=\frac{1}{2}(\alpha \beta-\beta \alpha) \beta \beta(\alpha \beta-\beta \alpha)$

$\phi_{8}=T^{+1} S^{0} S^{0}=\frac{1}{2} \alpha \alpha(\alpha \beta-\beta \alpha)(\alpha \beta-\beta \alpha)$

$\phi_{9}=\mathrm{T}^{0} \mathrm{~S}^{0} \mathrm{~S}^{0}=\frac{1}{2 \sqrt{2}}(\alpha \beta+\beta \alpha)(\alpha \beta-\beta \alpha)(\alpha \beta-\beta \alpha)$

$\phi_{10}=\mathrm{T}^{-1} \mathrm{~S}^{0} \mathrm{~S}^{0}=\frac{1}{2} \beta \beta(\alpha \beta-\beta \alpha)(\alpha \beta-\beta \alpha)$

27 singlet-triplet-triplet trimers(STT):

$\phi_{11}=\mathrm{S}^{0} \mathrm{~T}^{+1} \mathrm{~T}^{+1}=\frac{1}{\sqrt{2}}(\alpha \beta-\beta \alpha) \alpha \alpha \alpha \alpha$

$\phi_{12}=\mathrm{S}^{0} \mathrm{~T}^{+1} \mathrm{~T}^{0}=\frac{1}{2}(\alpha \beta-\beta \alpha) \alpha \alpha(\alpha \beta+\beta \alpha)$

$\phi_{13}=\mathrm{S}^{0} \mathrm{~T}^{+1} \mathrm{~T}^{-1}=\frac{1}{\sqrt{2}}(\alpha \beta-\beta \alpha) \alpha \alpha \beta \beta$ 


$$
\begin{aligned}
& \phi_{14}=\mathrm{S}^{0} \mathrm{~T}^{0} \mathrm{~T}^{+1}=\frac{1}{2}(\alpha \beta-\beta \alpha)(\alpha \beta+\beta \alpha) \alpha \alpha \\
& \phi_{15}=S^{0} \mathrm{~T}^{0} \mathrm{~T}^{0}=\frac{1}{2 \sqrt{2}}(\alpha \beta-\beta \alpha)(\alpha \beta+\beta \alpha)(\alpha \beta+\beta \alpha) \\
& \phi_{16}=\mathrm{S}^{0} \mathrm{~T}^{0} \mathrm{~T}^{-1}=\frac{1}{2}(\alpha \beta-\beta \alpha)(\alpha \beta+\beta \alpha) \beta \beta \\
& \phi_{17}=\mathrm{S}^{0} \mathrm{~T}^{-1} \mathrm{~T}^{+1}=\frac{1}{\sqrt{2}}(\alpha \beta-\beta \alpha) \beta \beta \alpha \alpha \\
& \phi_{18}=\mathrm{S}^{0} \mathrm{~T}^{-1} \mathrm{~T}^{0}=\frac{1}{2}(\alpha \beta-\beta \alpha) \beta \beta(\alpha \beta+\beta \alpha) \\
& \phi_{19}=\mathrm{S}^{0} \mathrm{~T}^{-1} \mathrm{~T}^{-1}=\frac{1}{\sqrt{2}}(\alpha \beta-\beta \alpha) \beta \beta \beta \beta \\
& \phi_{20}=\mathrm{T}^{+1} \mathrm{~S}^{0} \mathrm{~T}^{+1}=\frac{1}{\sqrt{2}} \alpha \alpha(\alpha \beta-\beta \alpha) \alpha \alpha \\
& \phi_{21}=\mathrm{T}^{+1} \mathrm{~S}^{0} \mathrm{~T}^{0}=\frac{1}{2} \alpha \alpha(\alpha \beta-\beta \alpha)(\alpha \beta+\beta \alpha) \\
& \phi_{22}=\mathrm{T}^{+1} \mathrm{~S}^{0} \mathrm{~T}^{-1}=\frac{1}{\sqrt{2}} \alpha \alpha(\alpha \beta-\beta \alpha) \beta \beta \\
& \phi_{23}=\mathrm{T}^{0} \mathrm{~S}^{0} \mathrm{~T}^{+1}=\frac{1}{2}(\alpha \beta+\beta \alpha)(\alpha \beta-\beta \alpha) \alpha \alpha \\
& \phi_{24}=\mathrm{T}^{0} \mathrm{~S}^{0} \mathrm{~T}^{0}=\frac{1}{2 \sqrt{2}}(\alpha \beta+\beta \alpha)(\alpha \beta-\beta \alpha)(\alpha \beta+\beta \alpha) \\
& \phi_{25}=\mathrm{T}^{0} \mathrm{~S}^{0} \mathrm{~T}^{-1}=\frac{1}{2}(\alpha \beta+\beta \alpha)(\alpha \beta-\beta \alpha) \beta \beta \\
& \phi_{26}=\mathrm{T}^{-1} \mathrm{~S}^{0} \mathrm{~T}^{+1}=\frac{1}{\sqrt{2}} \beta \beta(\alpha \beta-\beta \alpha) \alpha \alpha \\
& \phi_{27}=\mathrm{T}^{-1} \mathrm{~S}^{0} \mathrm{~T}^{0}=\frac{1}{2} \beta \beta(\alpha \beta-\beta \alpha)(\alpha \beta+\beta \alpha) \\
& \phi_{28}=\mathrm{T}^{-1} \mathrm{~S}^{0} \mathrm{~T}^{-1}=\frac{1}{\sqrt{2}} \beta \beta(\alpha \beta-\beta \alpha) \beta \beta \\
& \phi_{29}=\mathrm{T}^{+1} \mathrm{~T}^{+1} \mathrm{~S}^{0}=\frac{1}{\sqrt{2}} \alpha \alpha \alpha \alpha(\alpha \beta-\beta \alpha) \\
& \phi_{30}=\mathrm{T}^{+1} \mathrm{~T}^{0} \mathrm{~S}^{0}=\frac{1}{2} \alpha \alpha(\alpha \beta+\beta \alpha)(\alpha \beta-\beta \alpha) \\
& \phi_{31}=\mathrm{T}^{+1} \mathrm{~T}^{-1} \mathrm{~S}^{0}=\frac{1}{\sqrt{2}} \alpha \alpha \beta \beta(\alpha \beta-\beta \alpha) \\
& \phi_{32}=\mathrm{T}^{0} \mathrm{~T}^{+1} \mathrm{~S}^{0}=\frac{1}{2}(\alpha \beta+\beta \alpha) \alpha \alpha(\alpha \beta-\beta \alpha) \\
& \phi_{33}=\mathrm{T}^{0} \mathrm{~T}^{0} \mathrm{~S}^{0}=\frac{1}{2 \sqrt{2}}(\alpha \beta+\beta \alpha)(\alpha \beta+\beta \alpha)(\alpha \beta-\beta \alpha) \\
& \phi_{34}=\mathrm{T}^{0} \mathrm{~T}^{-1} \mathrm{~S}^{0}=\frac{1}{2}(\alpha \beta+\beta \alpha) \beta \beta(\alpha \beta-\beta \alpha) \\
& \phi_{35}=\mathrm{T}^{-1} \mathrm{~T}^{+1} \mathrm{~S}^{0}=\frac{1}{\sqrt{2}} \beta \beta \alpha \alpha(\alpha \beta-\beta \alpha)
\end{aligned}
$$


$\phi_{36}=\mathrm{T}^{-1} \mathrm{~T}^{0} \mathrm{~S}^{0}=\frac{1}{2} \beta \beta(\alpha \beta+\beta \alpha)(\alpha \beta-\beta \alpha)$

$\phi_{37}=\mathrm{T}^{-1} \mathrm{~T}^{-1} \mathrm{~S}^{0}=\frac{1}{\sqrt{2}} \beta \beta \beta \beta(\alpha \beta-\beta \alpha)$

and 27 triplet-triplet-triplet timers(TTT):

$\phi_{38}=\mathrm{T}^{+1} \mathrm{~T}^{+1} \mathrm{~T}^{+1}=\alpha \alpha \alpha \alpha \alpha \alpha$

$\phi_{39}=\mathrm{T}^{+1} \mathrm{~T}^{+1} \mathrm{~T}^{0}=\frac{1}{\sqrt{2}} \alpha \alpha \alpha \alpha(\alpha \beta+\beta \alpha)$

$\phi_{40}=\mathrm{T}^{+1} \mathrm{~T}^{+1} \mathrm{~T}^{-1}=\alpha \alpha \alpha \alpha \beta \beta$

$\phi_{41}=\mathrm{T}^{+1} \mathrm{~T}^{0} \mathrm{~T}^{+1}=\frac{1}{\sqrt{2}} \alpha \alpha(\alpha \beta+\beta \alpha) \alpha \alpha$

$\phi_{42}=\mathrm{T}^{+1} \mathrm{~T}^{0} \mathrm{~T}^{0}=\frac{1}{2} \alpha \alpha(\alpha \beta+\beta \alpha)(\alpha \beta+\beta \alpha)$

(S1ap)

$\phi_{43}=\mathrm{T}^{+1} \mathrm{~T}^{0} \mathrm{~T}^{-1}=\frac{1}{\sqrt{2}} \alpha \alpha(\alpha \beta+\beta \alpha) \beta \beta$

$\phi_{44}=\mathrm{T}^{+1} \mathrm{~T}^{-1} \mathrm{~T}^{+1}=\alpha \alpha \beta \beta \alpha \alpha$

$\phi_{45}=\mathrm{T}^{+1} \mathrm{~T}^{-1} \mathrm{~T}^{0}=\frac{1}{\sqrt{2}} \alpha \alpha \beta \beta(\alpha \beta+\beta \alpha)$

$\phi_{46}=\mathrm{T}^{+1} \mathrm{~T}^{-1} \mathrm{~T}^{-1}=\alpha \alpha \beta \beta \beta \beta$

$\phi_{47}=\mathrm{T}^{0} \mathrm{~T}^{+1} \mathrm{~T}^{+1}=\frac{1}{\sqrt{2}}(\alpha \beta+\beta \alpha) \alpha \alpha \alpha \alpha$

$\phi_{48}=\mathrm{T}^{0} \mathrm{~T}^{+1} \mathrm{~T}^{0}=\frac{1}{2}(\alpha \beta+\beta \alpha) \alpha \alpha(\alpha \beta+\beta \alpha)$

$\phi_{49}=\mathrm{T}^{0} \mathrm{~T}^{+1} \mathrm{~T}^{-1}=\frac{1}{\sqrt{2}}(\alpha \beta+\beta \alpha) \alpha \alpha \beta \beta$

$\phi_{50}=\mathrm{T}^{0} \mathrm{~T}^{0} \mathrm{~T}^{+1}=\frac{1}{2}(\alpha \beta+\beta \alpha)(\alpha \beta+\beta \alpha) \alpha \alpha$

$\phi_{51}=\mathrm{T}^{0} \mathrm{~T}^{0} \mathrm{~T}^{0}=\frac{1}{2 \sqrt{2}}(\alpha \beta+\beta \alpha)(\alpha \beta+\beta \alpha)(\alpha \beta+\beta \alpha)$

(S1ay)

$\phi_{52}=\mathrm{T}^{0} \mathrm{~T}^{0} \mathrm{~T}^{-1}=\frac{1}{2}(\alpha \beta+\beta \alpha)(\alpha \beta+\beta \alpha) \beta \beta$

$\phi_{53}=\mathrm{T}^{0} \mathrm{~T}^{-1} \mathrm{~T}^{+1}=\frac{1}{\sqrt{2}}(\alpha \beta+\beta \alpha) \beta \beta \alpha \alpha$

$\phi_{54}=\mathrm{T}^{0} \mathrm{~T}^{-1} \mathrm{~T}^{0}=\frac{1}{2}(\alpha \beta+\beta \alpha) \beta \beta(\alpha \beta+\beta \alpha)$

$\phi_{55}=\mathrm{T}^{0} \mathrm{~T}^{-1} \mathrm{~T}^{-1}=\frac{1}{\sqrt{2}}(\alpha \beta+\beta \alpha) \beta \beta \beta \beta$

$\phi_{56}=\mathrm{T}^{-1} \mathrm{~T}^{+1} \mathrm{~T}^{+1}=\beta \beta \alpha \alpha \alpha \alpha$

$\phi_{57}=\mathrm{T}^{-1} \mathrm{~T}^{+1} \mathrm{~T}^{0}=\frac{1}{\sqrt{2}} \beta \beta \alpha \alpha(\alpha \beta+\beta \alpha)$

$\phi_{58}=\mathrm{T}^{-1} \mathrm{~T}^{+1} \mathrm{~T}^{-1}=\beta \beta \alpha \alpha \beta \beta$

$\phi_{59}=\mathrm{T}^{-1} \mathrm{~T}^{0} \mathrm{~T}^{+1}=\frac{1}{\sqrt{2}} \beta \beta(\alpha \beta+\beta \alpha) \alpha \alpha$ 


$$
\begin{aligned}
& \phi_{60}=\mathrm{T}^{-1} \mathrm{~T}^{0} \mathrm{~T}^{0}=\frac{1}{2} \beta \beta(\alpha \beta+\beta \alpha)(\alpha \beta+\beta \alpha) \\
& \phi_{61}=\mathrm{T}^{-1} \mathrm{~T}^{0} \mathrm{~T}^{-1}=\frac{1}{\sqrt{2}} \beta \beta(\alpha \beta+\beta \alpha) \beta \beta \\
& \phi_{62}=\mathrm{T}^{-1} \mathrm{~T}^{-1} \mathrm{~T}^{+1}=\beta \beta \beta \beta \alpha \alpha \\
& \phi_{63}=\mathrm{T}^{-1} \mathrm{~T}^{-1} \mathrm{~T}^{0}=\frac{1}{\sqrt{2}} \beta \beta \beta \beta(\alpha \beta+\beta \alpha) \\
& \phi_{64}=\mathrm{T}^{-1} \mathrm{~T}^{-1} \mathrm{~T}^{-1}=\beta \beta \beta \beta \beta \beta
\end{aligned}
$$

2. Functions $\mathrm{S} 2 \mathrm{a}$ to $\mathrm{S} 2 \mathrm{t}$ refer to 64 spin eigenfunctions in $6 \times 6$ active space, the functions are written according to different multiplets, i.e., $\psi_{\mathrm{T}_{2}{ }^{+1}}$ refers to a second triplet eigenfunction , and the value of it in $\mathrm{Z}$ projection is +1 .

SSS:

$\left|\psi_{1}\right\rangle=\left|\psi_{S_{1}^{0}}\right\rangle=\phi_{1} ;$

singlet (S2a)

SST:

$$
\begin{aligned}
& \left|\psi_{2}\right\rangle=\left|\psi_{\mathrm{T}_{1}^{+1}}\right\rangle=\frac{1}{\sqrt{2}}\left(\phi_{2}-\phi_{8}\right), \\
& \left|\psi_{3}\right\rangle=\left|\Psi_{\mathrm{T}_{1}^{0}}\right\rangle=\frac{1}{\sqrt{2}}\left(\phi_{3}-\phi_{9}\right), \\
& \left|\psi_{4}\right\rangle=\left|\Psi_{\mathrm{T}_{1}^{-1}}\right\rangle=\frac{1}{\sqrt{2}}\left(\phi_{4}-\phi_{10}\right) ; \\
& \left|\psi_{5}\right\rangle=\left|\psi_{\mathrm{T}_{2}+1}\right\rangle=\frac{1}{\sqrt{2}}\left(\phi_{2}+\phi_{8}\right), \\
& \left|\psi_{6}\right\rangle=\left|\psi_{T_{2}^{0}}\right\rangle=\frac{1}{\sqrt{2}}\left(\phi_{3}+\phi_{9}\right), \\
& \left|\psi_{7}\right\rangle=\left|\psi_{\mathrm{T}_{2}^{-1}}\right\rangle=\frac{1}{\sqrt{2}}\left(\phi_{4}+\phi_{10}\right) ; \\
& \left|\psi_{8}\right\rangle=\left|\psi_{\mathrm{T}_{3}^{+1}}\right\rangle=\phi_{5}, \\
& \left|\psi_{9}\right\rangle=\left|\psi_{\mathrm{T}_{3}^{0}}\right\rangle=\phi_{6} \text {, } \\
& \left|\psi_{10}\right\rangle=\left|\psi_{\mathrm{T}_{3}-1}\right\rangle=\phi_{7} \text {; }
\end{aligned}
$$

STT:

$$
\begin{aligned}
& \left|\Psi_{11}\right\rangle=\left|\Psi_{Q_{1}^{+2}}\right\rangle=\frac{1}{\sqrt{2}}\left(\phi_{11}+\phi_{29}\right), \\
& \left|\Psi_{12}\right\rangle=\left|\Psi_{Q_{1}^{+1}}\right\rangle=\frac{1}{2}\left(\phi_{12}+\phi_{14}+\phi_{30}+\phi_{32}\right), \\
& \left|\Psi_{13}\right\rangle=\left|\Psi_{Q_{1}^{0}}\right\rangle=\frac{1}{\sqrt{12}}\left(\phi_{13}+\phi_{17}+2 \phi_{15}+\phi_{31}+\phi_{35}+2 \phi_{33}\right), \\
& \left|\Psi_{14}\right\rangle=\left|\Psi_{Q_{1}^{-1}}\right\rangle=\frac{1}{2}\left(\phi_{16}+\phi_{18}+\phi_{34}+\phi_{36}\right), \\
& \left|\Psi_{15}\right\rangle=\left|\Psi_{Q_{1}^{-2}}\right\rangle=\frac{1}{\sqrt{2}}\left(\phi_{19}+\phi_{37}\right), \\
& \left|\Psi_{16}\right\rangle=\left|\Psi_{Q_{2}^{+2}}\right\rangle=\phi_{20} ;
\end{aligned}
$$




$$
\begin{aligned}
& \left|\Psi_{17}\right\rangle=\left|\Psi_{Q_{2}^{+1}}\right\rangle=\frac{1}{\sqrt{2}}\left(\phi_{21}+\phi_{23}\right) \\
& \left|\Psi_{18}\right\rangle=\left|\Psi_{Q_{2}^{0}}\right\rangle=\frac{1}{\sqrt{6}}\left(\phi_{22}+\phi_{26}+2 \phi_{24}\right) \text {, } \\
& \left|\psi_{19}\right\rangle=\left|\Psi_{Q_{2}^{-1}}\right\rangle=\frac{1}{\sqrt{2}}\left(\phi_{25}+\phi_{27}\right), \\
& \left|\Psi_{20}\right\rangle=\left|\Psi_{Q_{2}^{0}}\right\rangle=\phi_{28} \\
& \left|\psi_{21}\right\rangle=\left|\psi_{Q_{3}^{+2}}\right\rangle=\frac{1}{\sqrt{2}}\left(\phi_{11}-\phi_{29}\right) \text {, } \\
& \left|\Psi_{22}\right\rangle=\left|\Psi_{Q_{3}^{+1}}\right\rangle=\frac{1}{2}\left(\phi_{12}+\phi_{14}-\phi_{30}-\phi_{32}\right), \\
& \left|\Psi_{23}\right\rangle=\left|\Psi_{Q_{3}^{0}}\right\rangle=\frac{1}{\sqrt{12}}\left(\phi_{13}+\phi_{17}+2 \phi_{15}-\phi_{31}-\phi_{35}-2 \phi_{33}\right), \\
& \left|\Psi_{24}\right\rangle=\left|\Psi_{Q_{3}^{-1}}\right\rangle=\frac{1}{2}\left(\phi_{16}+\phi_{18}-\phi_{34}-\phi_{36}\right) \text {, } \\
& \left|\Psi_{25}\right\rangle=\left|\Psi_{Q_{3}^{-2}}\right\rangle=\frac{1}{\sqrt{2}}\left(\phi_{19}-\phi_{37}\right) \\
& \left|\Psi_{26}\right\rangle=\left|\Psi_{\mathrm{T}_{4}^{+1}}\right\rangle=\frac{1}{2}\left(\phi_{12}+\phi_{30}-\phi_{14}-\phi_{32}\right), \\
& \left|\Psi_{27}\right\rangle=\left|\Psi_{T_{4}^{0}}\right\rangle=\frac{1}{2}\left(\phi_{13}+\phi_{31}-\phi_{17}-\phi_{35}\right), \\
& \left|\Psi_{28}\right\rangle=\left|\Psi_{\mathrm{T}_{4}^{-1}}\right\rangle=\frac{1}{2}\left(\phi_{16}+\phi_{36}-\phi_{18}-\phi_{34}\right) ; \\
& \left|\psi_{29}\right\rangle=\left|\Psi_{T_{5}^{+1}}\right\rangle=\frac{1}{\sqrt{2}}\left(\phi_{21}-\phi_{23}\right), \\
& \left|\Psi_{30}\right\rangle=\left|\Psi_{T_{5}^{0}}\right\rangle=\frac{1}{\sqrt{2}}\left(\phi_{22}-\phi_{26}\right) \text {, } \\
& \left|\Psi_{31}\right\rangle=\left|\Psi_{T_{5}-1}\right\rangle=\frac{1}{\sqrt{2}}\left(\phi_{25}-\phi_{27}\right) ; \\
& \left|\Psi_{32}\right\rangle=\left|\Psi_{\mathrm{T}_{6}^{+1}}\right\rangle=\frac{1}{2}\left(\phi_{12}-\phi_{30}-\phi_{14}+\phi_{32}\right), \\
& \left|\psi_{33}\right\rangle=\left|\psi_{\mathrm{T}_{6}^{0}}\right\rangle=\frac{1}{2}\left(\phi_{13}-\phi_{31}-\phi_{17}+\phi_{35}\right), \\
& \left|\Psi_{34}\right\rangle=\left|\Psi_{\mathrm{T}_{6}^{-1}}\right\rangle=\frac{1}{2}\left(\phi_{16}-\phi_{36}-\phi_{18}+\phi_{34}\right) ; \\
& \left|\Psi_{35}\right\rangle=\left|\Psi_{S_{2}^{0}}\right\rangle=\frac{1}{\sqrt{6}}\left(\phi_{13}+\phi_{17}-\phi_{15}+\phi_{31}+\phi_{35}-\phi_{33}\right) ; \\
& \left|\psi_{36}\right\rangle=\left|\Psi_{S_{3}^{0}}\right\rangle=\frac{1}{\sqrt{3}}\left(\phi_{22}+\phi_{26}-\phi_{24}\right) ; \\
& \left|\Psi_{37}\right\rangle=\left|\Psi_{S_{4}}\right\rangle=\frac{1}{\sqrt{6}}\left(\phi_{13}+\phi_{17}-\phi_{15}-\phi_{31}-\phi_{35}+\phi_{33}\right) ; \\
& \text { quintet (S2f) } \\
& \text { quintet (S2g) } \\
& \text { triplet (S3h) } \\
& \text { singlet (S2m) }
\end{aligned}
$$




$$
\begin{aligned}
& \left|\Psi_{39}\right\rangle=\left|\Psi_{\mathrm{Sep}_{4}{ }^{+2}}\right\rangle=\frac{1}{\sqrt{3}}\left(\phi_{39}+\phi_{41}+\phi_{47}\right) \text {, } \\
& \left|\psi_{40}\right\rangle=\left|\Psi_{\mathrm{Sep}_{4}{ }^{+1}}\right\rangle=\frac{1}{\sqrt{15}}\left(2 \phi_{42}+2 \phi_{48}+2 \phi_{50}+\phi_{40}+\phi_{44}+\phi_{56}\right) \\
& \left|\psi_{41}\right\rangle=\left|\psi_{\mathrm{Sep}_{4}^{0}}\right\rangle=\frac{1}{\sqrt{10}}\left(\phi_{43}+\phi_{59}+\phi_{45}+\phi_{57}+\phi_{49}+\phi_{53}+2 \phi_{51}\right), \\
& \left|\psi_{42}\right\rangle=\left|\psi_{\mathrm{Sep}_{4}{ }^{-1}}\right\rangle=\frac{1}{\sqrt{15}}\left(2 \phi_{52}+2 \phi_{54}+2 \phi_{60}+\phi_{46}+\phi_{58}+\phi_{62}\right), \\
& \left|\psi_{43}\right\rangle=\left|\psi_{\text {Sep }_{4}-2}\right\rangle=\frac{1}{\sqrt{3}}\left(\phi_{55}+\phi_{61}+\phi_{63}\right), \\
& \left|\psi_{44}\right\rangle=\left|\Psi_{\mathrm{Sep}_{4}^{-3}}\right\rangle=\phi_{64} \text {; } \\
& \left|\psi_{45}\right\rangle=\left|\psi_{Q_{4}^{+2}}\right\rangle=\frac{1}{\sqrt{2}}\left(\phi_{39}-\phi_{47}\right) \text {, } \\
& \left|\Psi_{46}\right\rangle=\left|\psi_{Q_{4}{ }^{+1}}\right\rangle=\frac{1}{2}\left(\phi_{42}-\phi_{50}+\phi_{40}-\phi_{56}\right), \\
& \left|\psi_{47}\right\rangle=\left|\Psi_{Q_{4}^{0}}\right\rangle=\frac{1}{\sqrt{12}}\left(\phi_{49}+\phi_{45}+2 \phi_{43}-\phi_{53}-\phi_{57}-2 \phi_{59}\right), \\
& \left|\Psi_{48}\right\rangle=\left|\Psi_{Q_{4}^{-1}}\right\rangle=\frac{1}{2}\left(\phi_{52}-\phi_{60}+\phi_{46}-\phi_{62}\right), \\
& \left|\psi_{49}\right\rangle=\left|\Psi_{Q_{4}^{-2}}\right\rangle=\frac{1}{\sqrt{2}}\left(\phi_{55}-\phi_{63}\right) \\
& \left|\psi_{50}\right\rangle=\left|\psi_{Q_{5}^{+2}}\right\rangle=\frac{1}{\sqrt{6}}\left(\phi_{39}+\phi_{47}-2 \phi_{41}\right), \\
& \left|\Psi_{51}\right\rangle=\left|\Psi_{Q_{5}^{+1}}\right\rangle=\frac{1}{\sqrt{12}}\left(2 \phi_{48}-\phi_{42}-\phi_{50}+\phi_{40}+\phi_{56}-2 \phi_{44}\right), \\
& \left|\psi_{52}\right\rangle=\left|\psi_{Q_{5}^{0}}\right\rangle=\frac{1}{2}\left(\phi_{57}-\phi_{45}+\phi_{49}-\phi_{53}\right), \\
& \left|\psi_{53}\right\rangle=\left|\Psi_{Q^{-1}}\right\rangle=\frac{1}{\sqrt{12}}\left(\phi_{52}+\phi_{60}-2 \phi_{54}-\phi_{46}-\phi_{62}+2 \phi_{58}\right), \\
& \left|\Psi_{54}\right\rangle=\left|\Psi_{Q_{5}^{-2}}\right\rangle=\frac{1}{\sqrt{6}}\left(2 \phi_{61}-\phi_{55}-\phi_{63}\right) ; \\
& \left|\psi_{55}\right\rangle=\left|\Psi_{\mathrm{T}_{7}^{+1}}\right\rangle=\frac{1}{2}\left(\phi_{50}-\phi_{42}+\phi_{40}-\phi_{56}\right), \\
& \left|\psi_{56}\right\rangle=\left|\Psi_{\mathrm{T}_{7}^{0}}\right\rangle=\frac{1}{2}\left(\phi_{49}-\phi_{45}+\phi_{53}-\phi_{57}\right), \\
& \left|\psi_{57}\right\rangle=\left|\psi_{\mathrm{T}_{7}^{-1}}\right\rangle=\frac{1}{2}\left(\phi_{52}-\phi_{60}+\phi_{62}-\phi_{46}\right) ; \\
& \left|\psi_{58}\right\rangle=\left|\Psi_{T_{8}+1}\right\rangle=\frac{1}{\sqrt{8}}\left(-\phi_{42}-\phi_{50}+\phi_{40}+\phi_{56}+2 \phi_{44}\right), \\
& \left|\psi_{59}\right\rangle=\left|\Psi_{T_{8}^{0}}\right\rangle=\frac{1}{\sqrt{8}}\left(\phi_{49}+\phi_{45}+\phi_{53}+\phi_{57}-2 \phi_{51}\right) \text {, } \\
& \left|\Psi_{60}\right\rangle=\left|\Psi_{\mathrm{T}_{8}^{-1}}\right\rangle=\frac{1}{\sqrt{8}}\left(-\phi_{60}-\phi_{52}+\phi_{62}+\phi_{46}+2 \phi_{58}\right)
\end{aligned}
$$




$$
\begin{aligned}
& \left|\Psi_{61}\right\rangle=\left|\Psi_{T_{9}^{+1}}\right\rangle=\frac{1}{\sqrt{40}}\left(\phi_{42}-4 \phi_{48}+\phi_{50}+3 \phi_{40}-2 \phi_{44}+3 \phi_{56}\right) \\
& \left|\Psi_{62}\right\rangle=\left|\Psi_{T_{9}^{0}}\right\rangle=\frac{1}{\sqrt{40}}\left(2 \phi_{51}+\phi_{45}+\phi_{57}+\phi_{49}+\phi_{53}-4 \phi_{43}-4 \phi_{59}\right) \\
& \left|\Psi_{63}\right\rangle=\left|\Psi_{T_{9}^{-1}}\right\rangle=\frac{1}{\sqrt{40}}\left(\phi_{60}-\phi_{54}+\phi_{52}+3 \phi_{62}-2 \phi_{58}+3 \phi_{46}\right) \\
& \left|\Psi_{64}\right\rangle=\left|\Psi_{S_{5}^{0}}\right\rangle=\frac{1}{\sqrt{6}}\left(-\phi_{45}+\phi_{57}+\phi_{43}-\phi_{59}-\phi_{49}+\phi_{53}\right)
\end{aligned}
$$

triplet (S2s)

3. Submatrices S3a-d refer to the diagonal subspace in Figure 2, while $\mathrm{a}=\frac{1}{\sqrt{2}}, b=\frac{1}{\sqrt{6}}, c=\frac{1}{\sqrt{3}}$ $, d=\frac{1}{2}, e=\frac{1}{\sqrt{12}}, \mathrm{f}=\frac{1}{\sqrt{15}}, \mathrm{~g}=\frac{1}{\sqrt{10}}, \mathrm{~h}=\frac{1}{\sqrt{40}}, \mathrm{i}=\frac{1}{\sqrt{8}}$.

$A=(1)$

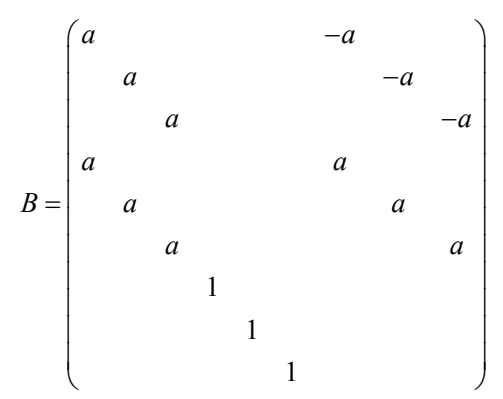

(S3b)

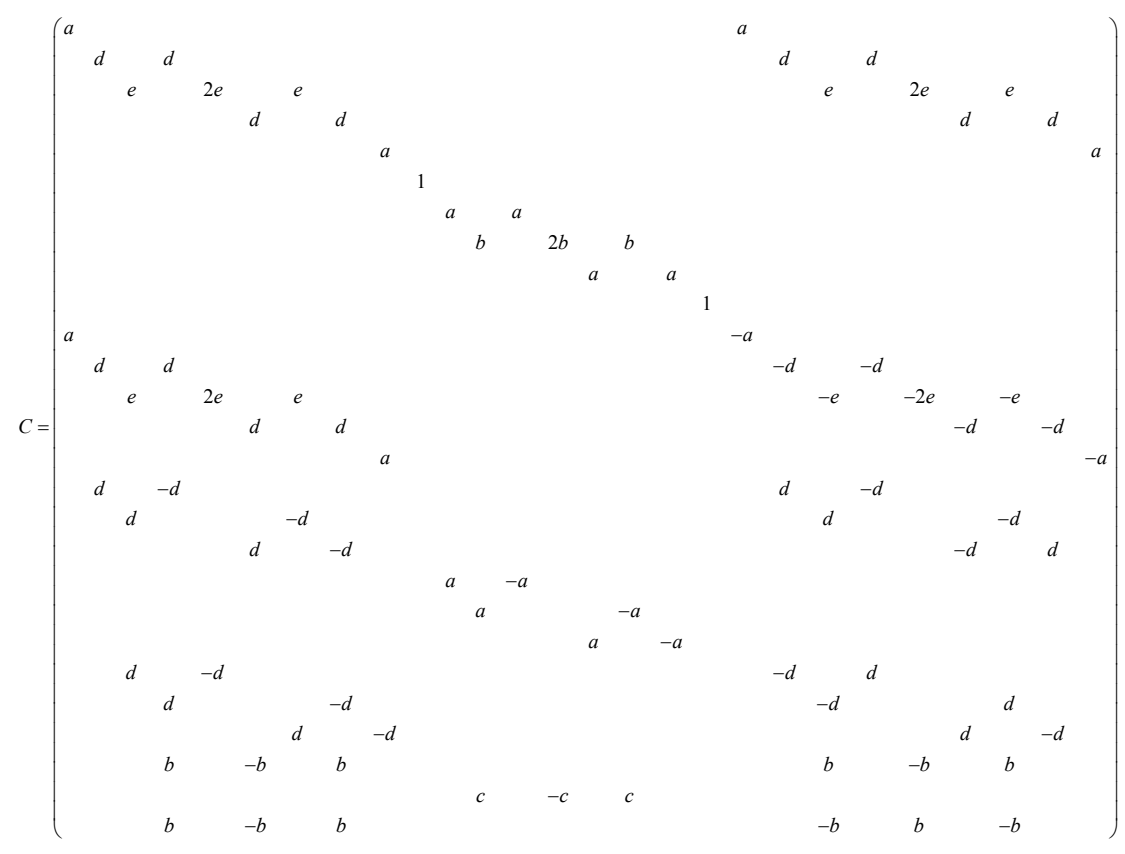

(S1c) 


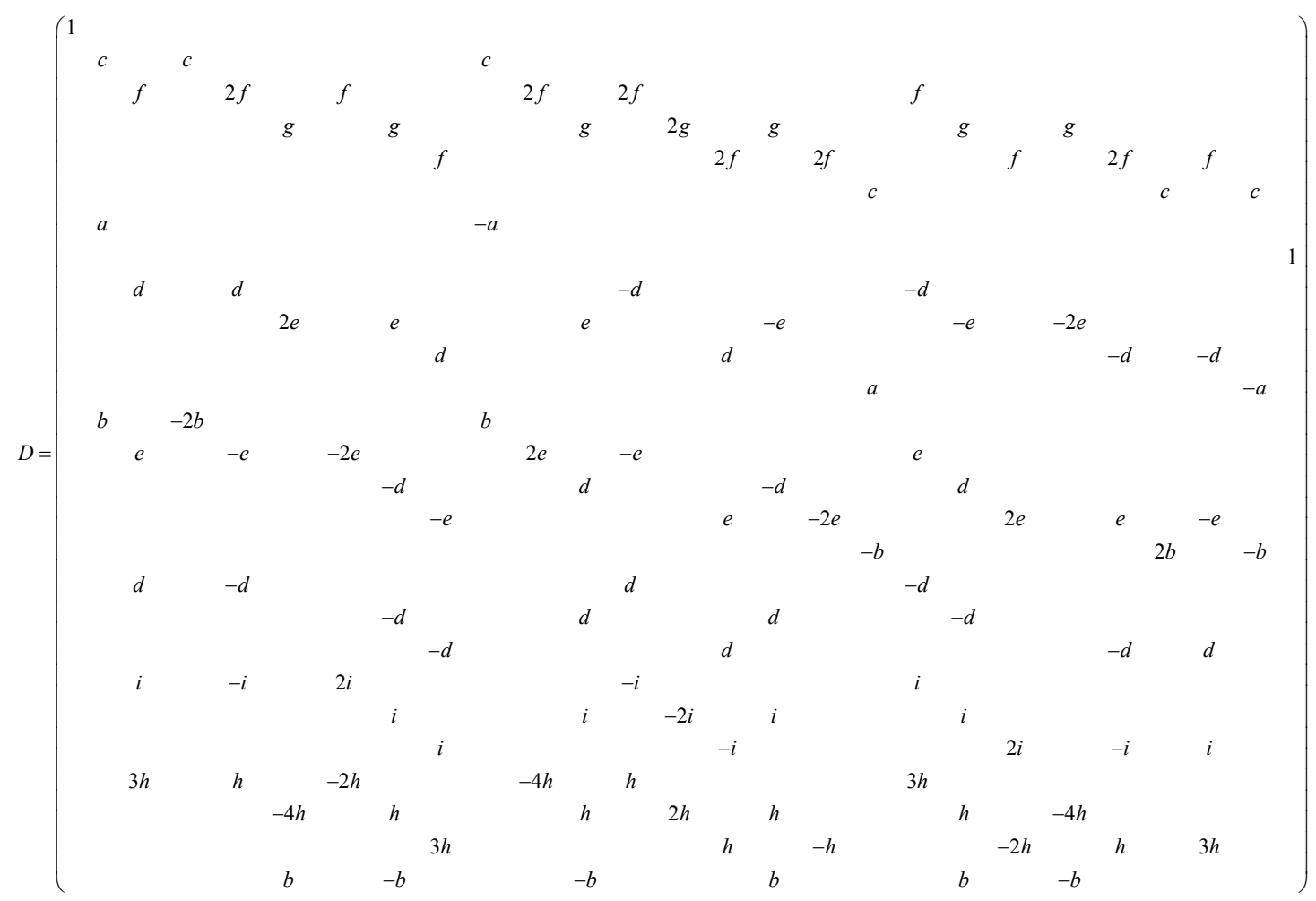

4. Exciton trimer state number.

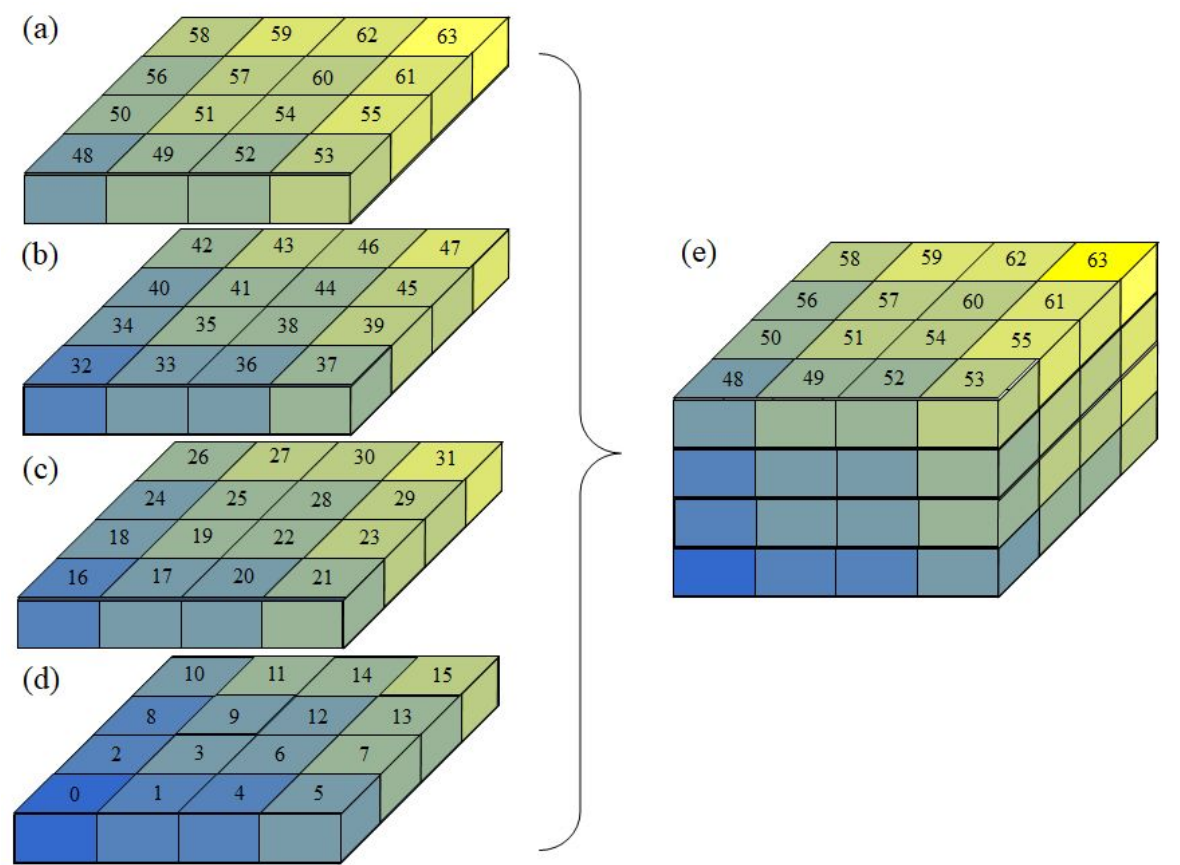

Figure S1. Figure 3, with each primitive spin function assigned a corresponding number, are shown in a different way. 
5. Energy levels of each multiplet. The energy spitted into several energy levels.

(a)

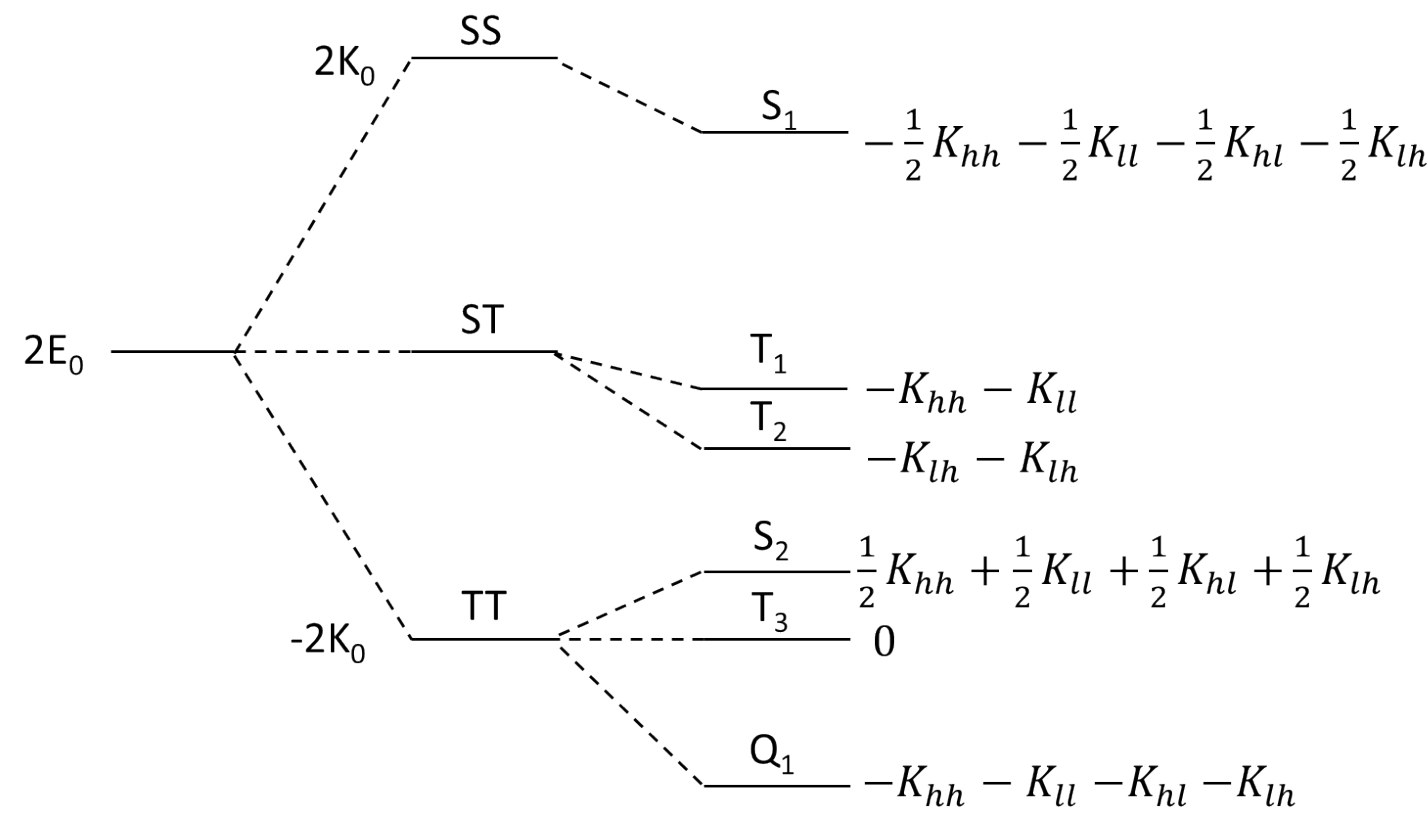

(b)

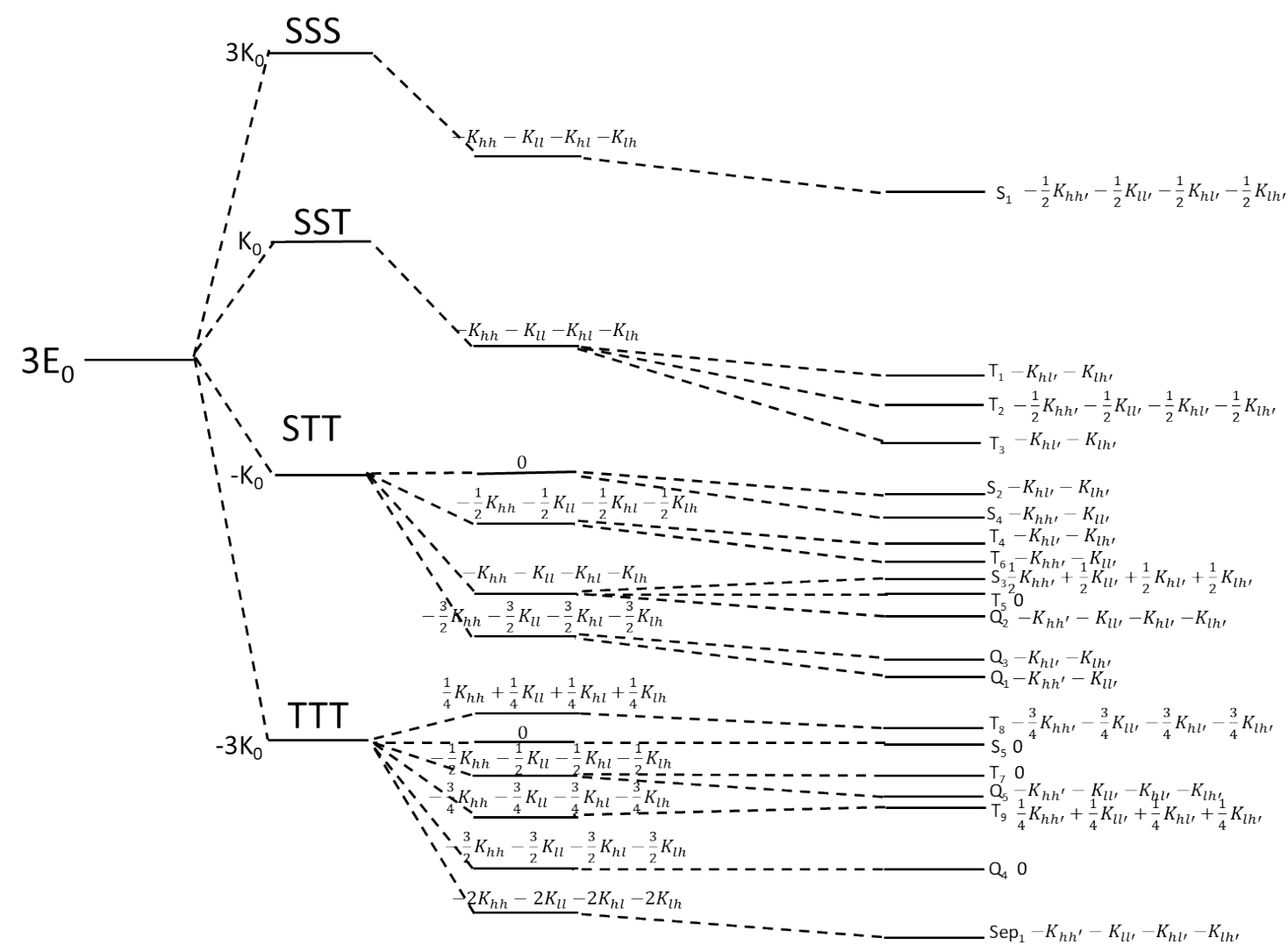

Figure S2. Assume $: \mathrm{K}_{0}>\mathrm{K}_{\mathrm{ll}}\left(\mathrm{K}_{\mathrm{hh}}\right)>\mathrm{K}_{\mathrm{lh}}\left(\mathrm{K}_{\mathrm{hl}}\right)>\mathrm{K}_{\mathrm{ll}}\left(\mathrm{K}_{\mathrm{hh}}\right)>\mathrm{K}_{\mathrm{lh}},\left(\mathrm{K}_{\mathrm{hl}}\right)$. (a) represents energy levels in dimer; (b) represents energy levels in trimer. 
6. Time dependent state populations of the model system for SF indunced stimulated emission.
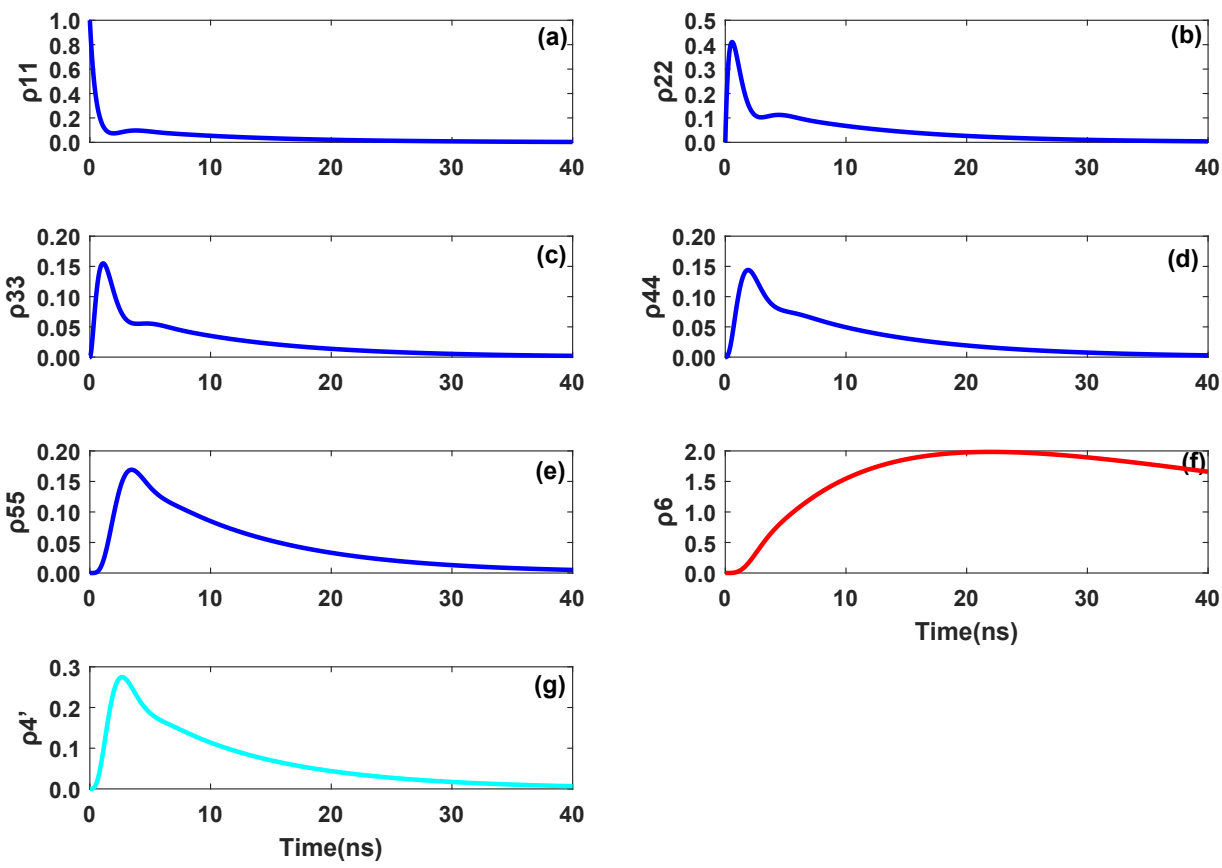

Figure S3. Time-dependent state populations and photon population of the model system for SF induced stimulated emission. States 1-5 (blue) are ground state, singlet exciton S1 state, triplet pair state, exciton trimer state, and transient state in the laser chromophore (see Figure 5). Separated triplet state (4') is in light blue, and photon population (6) is in red. 

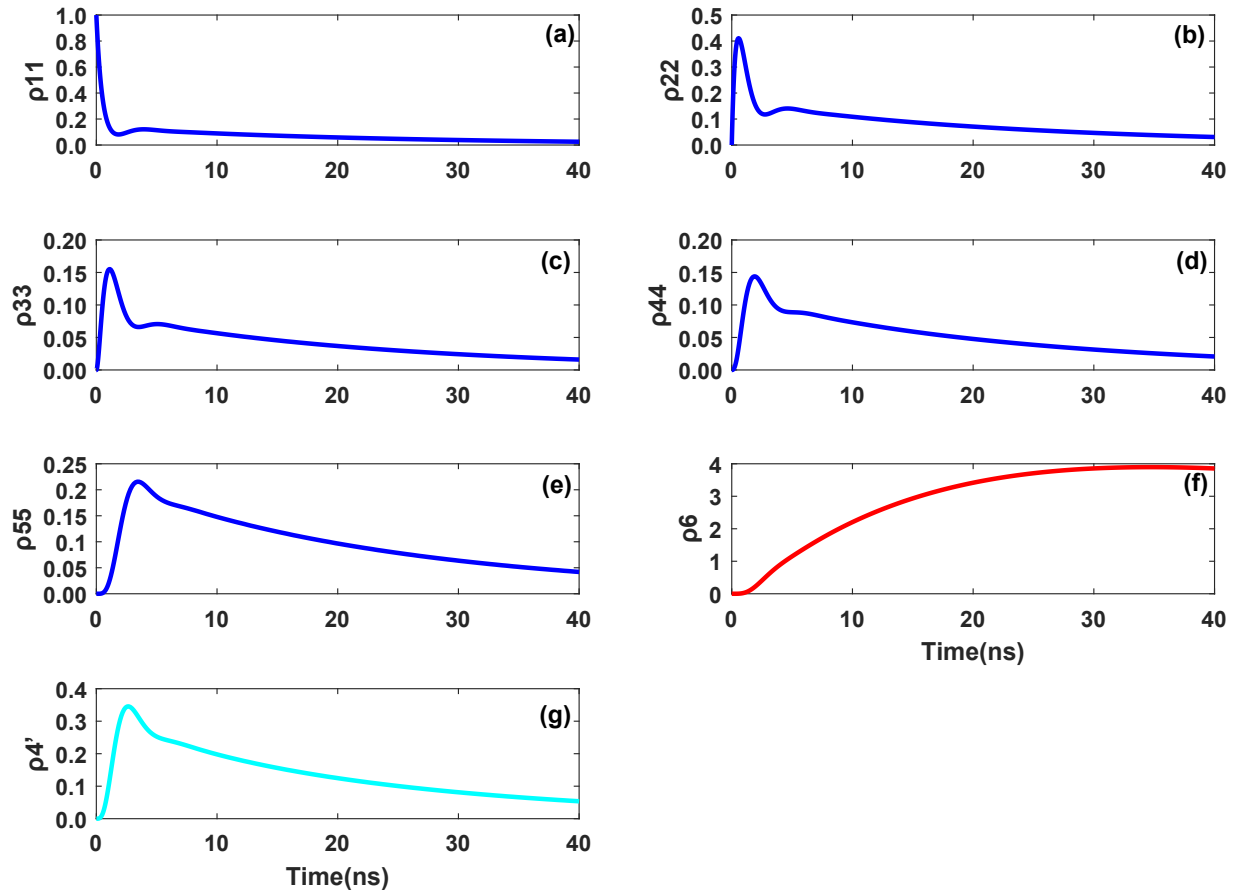

Figure S4. The same as Figure S3 but for the case B involving exciton tetramer. 